\title{
ANÁLISE DE ESTABILIDADE ASSINTÓTICA E EXPONENCIAL EM REDES NEURAIS ARTIFICIAIS SUJEITAS A RETARDO NO TEMPO E A INCERTEZAS DO TIPO POLITÓPICAS
}

\author{
Fernando de Oliveira Souza* \\ oliveiraecpdee.ufmg.br
}

\author{
Reinaldo Martinez Palhares* \\ palhares@cpdee.ufmg.br
}

\author{
Petr Yakovlevitch Ekel ${ }^{\dagger}$ \\ ekelepucminas.br \\ * Departamento de Engenharia Eletrônica \\ Universidade Federal de Minas Gerais \\ Av. Antônio Carlos 6627 - Pampulha \\ 31270-010, Belo Horizonte - MG - Brasil \\ ${ }^{\dagger}$ Programa de Pós-Graduação em Engenharia Elétrica \\ Pontifícia Universidade Católica de Minas Gerais \\ Av. Dom José Gaspar 500 - Coração Eucarístico \\ 30535-610, Belo Horizonte - MG - Brasil
}

\begin{abstract}
This paper presents sufficient conditions for asymptotic and exponential stability of a class of artificial neural networks (ANNs) subject to constant or time-varying delay and polytope-bounded uncertainties.
\end{abstract}

The proposed approach is based on Lyapunov-Krasovskii stability theory, and the linear matrix inequalities (LMIs) technique introducing slack matrices so that convex optimization algorithms can be used.

Three examples with numerical simulations are performed to demonstrate the effectiveness of the proposed method. The first example deals with the asymptotic stability analysis, the second one with the robust stability analysis and the last one with exponential stability.

\footnotetext{
Artigo submetido em 28/04/2006

1a. Revisão em 02/05/2007

2a. Revisão em 24/09/2007

3a. Revisão em 29/02/2008

Aceito sob recomendação do Editor Associado Prof. Liu Hsu
}

KEYWORDS: Lyapunov-Krasovskii Theory, Robust Asymptotic and Exponential Stability, Linear Matrix Inequality, Neural Networks, Time-Delay.

\section{RESUMO}

Este artigo apresenta condições suficientes para estabilidade assintótica e exponencial de uma classe de RNAs (Redes Neurais Artificiais) sujeitas a influência de retardo no tempo (constante ou variante) e/ou sujeitas a incertezas paramétricas do tipo politópicas.

A abordagem proposta é baseada na teoria de estabilidade de Lyapunov-Krasovskii, e utiliza desigualdades matriciais lineares (LMIs - do inglês, Linear Matrix Inequalities) introduzindo matrizes de relaxação, de modo que os resultados obtidos podem ser facilmente resolvidos por meio de algoritmos de otimização convexa.

Três exemplos com simulações numéricas são usados para demonstrar a eficiência do método proposto. O primeiro exemplo analisa a estabilidade assintótica, o segundo a es- 
tabilidade robusta e o último exemplo a estabilidade exponencial.

PALAVRAS-CHAVE: Teoria da Estabilidade de LyapunovKrasovskii, Estabilidade Robusta, Assintótica e Exponencial, Desigualdades Matriciais Lineares (LMIs - do inglês, Linear Matrix Inequalities), Redes Neurais Artificiais (RNAs), Retardo no Tempo.

\section{INTRODUÇÃO}

Este artigo aborda o estudo de uma classe de Redes Neurais Artificiais (RNAs), que podem ser vistas como sistemas dinâmicos. Especificamente, a classe de redes neurais recorrentes auto-associativas. Atualmente, é consenso que RNAs dinâmicas, tem um forte apelo em aplicações relacionadas a reconhecimento de padrões, problemas de otimização e a processamento de sinais e imagem.

Desde o trabalho pioneiro Hopfield (1982), o qual mostrou a relação entre redes neurais recorrentes auto-associativas e sistemas físicos, uma gama de novos trabalhos apareceram na literatura. Entretanto, quando RNAs são implementadas analogicamente, pode aparecer entre os canais de comunicação dos neurônios o retardo no tempo. Sabe-se que o retardo no tempo pode ocasionar efeitos na dinâmica e no processo de aprendizagem das RNAs, o que fortalece ainda mais um problema intrínseco de qualquer sistema dinâmico, que diz respeito a quesitos de estabilidade. Os leitores interessados em mais detalhes dos efeitos causados pelo retardo no tempo em RNAs, devem consultar a referência Baldi and Atiya (1994). Além disso, pode ser observado nos exemplos deste trabalho, especialmente no primeiro, como a incorporação do retardo no tempo influência a estabilidade das RNAs, o que pode causar fenômenos oscilatórios ou instabilidade.

O primeiro estudo da influência do retardo no tempo em RNAs foi em Marcus and Westervelt (1989), seguido por, Gopalsamy and He (1994a), que consideraram a introdução de diferentes retardos no tempo em diferentes canais de comunicação, e por Joy (1999), no qual foi adicionado ao modelo também, informação sobre a propagação instantânea dos sinais.

Com o aumento crescente de estudos nesta área, gerou-se na literatura dois conceitos sobre estabilidade em RNAs sujeitas a influência do retardo no tempo. O primeiro, que não inclui qualquer informação sobre o tamanho do retardo no tempo, é conhecido como independente do retardo no tempo: Marcus and Westervelt (1989), Roska et al. (1992), Roska et al. (1993), Gopalsamy and He (1994a), Gopalsamy and He (1994b), Ye et al. (1994), Zhang (1996), Liao and J.Yu (1998), den Driessche and Zou (1998), Liao and Yu (1998), Joy (1999), Joy (2000), Arik (2000), Cao (2000), Chen and
Rong (2003) e Wang et al. (2004). O outro conceito, leva em conta explicitamente o retardo no tempo na formulação do problema, sendo conhecido como dependente do retardo no tempo: Wei and Ruan (1999), Zhang et al. (2003), Chen et al. (2004), Li et al. (2004), Ensari and Arik (2005) e Zeng et al. (2005). Conseqüentemente, os métodos independentes do retardo no tempo, são adequados para tratarem apenas sistemas sujeitos a retardo no tempo incomensuráveis. E, por outro lado, os métodos dependentes do retardo no tempo permitem garantir a estabilidade do sistema, para determinados valores do retardo no tempo, portanto este método pode ser mais aplicável.

Com o objetivo de obter métodos menos conservadores e a necessidade de se obter condições viáveis para se verificar a estabilidade de RNAs em situações práticas, o uso das LMIs por suas potencialidades em termos de convexidade, manipulações e introdução de relaxações, tornou-se uma ferramenta com forte apelo, sendo tema de diversos trabalhos: Liao et al. (2002a), Liao et al. (2002b), Chen and Rong (2003), Singh (2004) e em Li et al. (2004).

Tratando-se de sistemas físicos, os quais usualmente sofrem de incertezas, as quais podem ser causadas por variações dos parâmetros, erros de modelamento, envelhecimento, ou por fatores ignorados, etc. O escopo deste trabalho também envolve redes neurais artificiais analógicas sujeitas a incertezas paramétricas do tipo politópicas.

Outro ponto do escopo deste trabalho diz respeito a estabilidade exponencial, que não é apenas um resultado teoricamente interessante. Pois, em termos práticos, a taxa exponencial de convergência é usada para determinar a velocidade de cômputos neurais, sujeitas a quaisquer transformações. Assim, é possível determinar uma estimativa do tempo em que a RNA leva para se estabilizar, sendo esse um índice de desempenho.

O objetivo deste artigo, é propor métodos de análise de estabilidade dependentes do retardo no tempo, para uma classe de RNAs, que podem apresentar parâmetros incertos e estarem sujeitas a retardo no tempo, constante ou variante. Neste trabalho desenvolvem-se abordagens para a análise da estabilidade assintótica e exponencial. O método proposto, parte de uma técnica apresentada em Wu et al. (2004), na qual matrizes de parâmetros livres são introduzidas nas LMIs, sem incluir qualquer dinâmica adicional ao sistema, permitindo que o método seja pouco conservador.

Finalmente a eficiência do método proposto é verificado por meio de três exemplos. Neste artigo " $T$ " sobrescrito representa transposto, $M>0(<0)$ significa que a matriz $M$ é definida positiva (negativa) e $\lambda_{\max }(\cdot)\left(\lambda_{\min }(\cdot)\right)$ denota o autovalor máximo (mínimo) do argumento $(\cdot)$. 


\section{PRELIMINARES}

John Hopfield (1982) mostrou que é possível relacionar redes neurais artificiais recorrentes com sistemas físicos, por meio de um "simples" circuito analógico, no qual, cada $i$-ésimo neurônio é representado por um circuito linear que consiste de um resistor $R_{i}$, um capacitor $C_{i}$, uma fonte de corrente $I_{i}$ e uma função de ativação não-linear de $g_{i}(\cdot)$. Entre o canal de comunicação de um $j$-ésimo neurônio e um $i$-ésimo neurônio há uma condutância $w_{j i}=1 / R_{j i}$ e os neurônios são conectados através de uma junção aditiva de corrente. Assim, aplicando a lei de Kirchoff das correntes nesse circuito, chega-se a seguinte equação diferencial,

$$
\begin{array}{r}
\frac{d v_{i}(t)}{d t}=-\frac{v_{i}(t)}{C_{i} R_{i}}+\frac{1}{C_{i}} \sum_{j=1}^{n} w_{i j} g_{i}\left(v_{i}(t)\right)+\frac{I_{i}}{C_{i}}, \\
i=1,2, \ldots, n .
\end{array}
$$

No trabalho pioneiro de Marcus and Westervelt (1989), introduziu-se um retardo no tempo constante $\bar{\tau}>0$ em (1), obtendo,

$$
\begin{array}{r}
\frac{d v_{i}(t)}{d t}=-\frac{v_{i}(t)}{C_{i} R_{i}}+\frac{1}{C_{i}} \sum_{j=1}^{n} w_{i j} g_{i}\left(v_{i}(t-\bar{\tau})\right)+\frac{I_{i}}{C_{i}}, \\
i=1,2, \ldots, n .
\end{array}
$$

No entanto, ao contrário do sistema descrito em (1), o sistema (2) tem um comportamento dinâmico mais realístico e complicado, devido a incorporação do retardo no tempo. Em Joy (1999) um novo termo foi considerado, de forma que, além do retardo no tempo na propagação dos sinais, o modelo da RNA inclui informação sobre a propagação instantânea dos sinais, sendo este um modelo mais geral,

$$
\begin{aligned}
& \frac{d v_{i}(t)}{d t}=-\frac{v_{i}(t)}{C_{i} R_{i}}+\frac{1}{C_{i}} \sum_{j=1}^{n} w_{i j}^{0} g_{i}\left(v_{i}(t)\right) \\
& +\frac{1}{C_{i}} \sum_{j=1}^{n} w_{i j}^{1} g_{i}\left(v_{i}(t-\bar{\tau})\right)+\frac{I_{i}}{C_{i}}, \quad i=1,2, \ldots, n .
\end{aligned}
$$

Outra dificuldade é que, ao contrário de apresentar um retardo constante, muitas redes neurais na prática podem estar sujeitas a retardo que varia no tempo. Dessa forma, " $v_{i}(t-$ $\bar{\tau})$ " em (3) é reescrito como " $v_{i}(t-d(t))$ ", obtendo-se,

$$
\begin{aligned}
& \frac{d v_{i}(t)}{d t}=-\frac{v_{i}(t)}{C_{i} R_{i}}+\frac{1}{C_{i}} \sum_{j=1}^{n} w_{i j}^{0} g_{i}\left(v_{i}(t)\right) \\
& +\frac{1}{C_{i}} \sum_{j=1}^{n} w_{i j}^{1} g_{i}\left(v_{i}(t-d(t))\right)+\frac{I_{i}}{C_{i}}, \quad i=1,2, \ldots, n .
\end{aligned}
$$

Neste trabalho, é considerado que o retardo variante no tempo $d(t)$, seja, diferenciável, positivo e limitado, portanto que satisfaça as seguintes condições,

$$
0<d(t) \leq \tau, \quad \dot{d}(t) \leq \mu<1,
$$

sendo $\tau$ e $\mu$ constantes.

Sabendo que, neste artigo são utilizadas abordagens do tipo LMI. O sistema (4) é reescrito de forma mais apropriada, fazendo as mudanças de variáveis: $a_{i}=1 /\left(R_{i} C_{i}\right)$, $i=1, \ldots, n, w_{i j}=w_{i j} / C_{i}, i, j=1, \ldots, n$ e $I_{i}=I_{i} / C_{i}$, $i=1, \ldots, n$.

Assim, a rede neural com retardo variante no tempo com $n$ neurônios é equivalente a:

$$
\frac{d v(t)}{d t}=-A v(t)+W_{0} g(v(t))+W_{1} g(v(t-d(t)))+I,
$$

na qual, $v(t)=\left[v_{1}(t), v_{2}(t), \ldots, v_{n}(t)\right]^{T} \in \mathbb{R}^{n}$ é o vetor de estados da rede neural, $A=\operatorname{diag}\left(a_{1}, a_{2}, \ldots, a_{n}\right)$ $\in \mathbb{R}^{n \times n}$ é uma matriz diagonal com elementos positivos, $a_{i}>0, W_{0}=\left(w_{i j}^{0}\right) \in \mathbb{R}^{n \times n}$ e $W_{1}=\left(w_{i j}^{1}\right)$ $\in \mathbb{R}^{n \times n}$ são pesos de conexão da matriz e os pesos de conexão da matriz com o retardo, respectivamente, $g(v(t))=$ $\left[g_{1}\left(v_{1}(t)\right), g_{2}\left(v_{2}(t)\right), \ldots, g_{n}\left(v_{n}(t)\right)\right]^{T} \in \mathbb{R}^{n}$ é a função de ativação dos neurônios com $g(0)=0$ e $I=\left[I_{1}, I_{2}, \ldots, I_{n}\right]^{T}$ $\in \mathbb{R}^{n}$ é um vetor constante.

Outra característica importante sobre a RNA em (6), diz respeito a existência e unicidade da solução. Portanto, para que essa RNA tenha uma solução e que esta seja única, certas restrições são impostas as suas funções de ativação, $g(v(\cdot))$. Para que exista uma solução, é suficiente que $g(v(\cdot))$ seja contínua em todos seus argumentos. Entretanto, esta restrição sozinha não garante a unicidade da solução. Portanto, para que isto aconteça, é imposto que $g(\cdot)$ satisfaça a condição:

$$
0 \leq \frac{g_{j}\left(v_{1}\right)-g_{j}\left(v_{2}\right)}{v_{1}-v_{2}} \leq \sigma_{j}, \quad \forall v_{1}, v_{2}, \quad \in \mathbb{R},
$$

$\operatorname{com} v_{1}, v_{2} \in \mathbb{R}, v_{1} \neq v_{2}, \sigma_{j} \in \mathbb{R}^{+}, g_{j}(0)=0$, $j=1,2, \ldots, n$. A demonstração dessa afirmação é encontrada em Zhang (2003). Além disso, note que a condição em (7) é Lipschitz.

Hipóteses do tipo setor para funções de ativação são usualmente consideradas em trabalhos da literatura, como em: Roska et al. (1992), Arik (2000), Cao (2000), Chen and Rong (2003), Zhang et al. (2003), Chen et al. (2004), Li et al. (2004), Ensari and Arik (2005) e Zeng et al. (2005). Além disso, para maiores detalhes sobre especificações e desempenho de diferentes funções de ativação, as seguintes referências são indicadas Morita (1993) e Morita and Amari (1993). 
A seguir, o ponto fixo, $v^{*}$, do sistema (6) é deslocado para a origem, por meio da transformação, $x=v-v^{*}$. Como resultado dessa transformação, é considerado o estudo das estabilidades assintótica e exponencial de RNAs com ponto fixo na origem. Sendo, o objeto de estudo ${ }^{1}$ deste artigo apresentado a seguir,

$$
\dot{x}(t)=-A x(t)+W_{0} f(x(t))+W_{1} f(x(t-d(t))),
$$

sendo que $x=\left[x_{1}, x_{2}, \ldots, x_{n}\right]^{T} \in \mathbb{R}^{n}$ é o vetor de estado do sistema transformado, e $f(x)=\left[f_{1}\left(x_{1}\right), f_{2}\left(x_{2}\right)\right.$, $\left.\ldots, f_{n}\left(x_{n}\right)\right]^{T} \in \mathbb{R}^{n} \operatorname{com} f_{i}\left(x_{i}\right)=g_{i}\left(x_{i}+v_{i}^{*}\right)-g_{i}\left(v_{i}^{*}\right)$, $i=1,2, \ldots, n$. Enquanto o retardo no tempo, $d(t)$, é variante, diferenciável, positivo e limitado.

Portando, considerando o sistema transformado em (8), a função de ativação $f(\cdot)$ é limitada, crescente e satisfaz a seguinte condição.

Hipótese (H) A função de ativação $f(\cdot)$ é limitada e satisfaz:

$$
0 \leq \frac{f_{j}(x)}{x} \leq \sigma_{j},
$$

sendo, $x \in \mathbb{R}, \sigma_{j} \in \mathbb{R}^{+}, f_{j}(0)=0, j=1,2, \ldots, n$.

Note que a condição anterior é obtida fazendo $v_{1}=x+v^{*} \mathrm{e}$ $v_{2}=v^{*}$ na condição em (7).

A seguir, algumas ferramentas matemáticas utilizadas são apresentadas. Os leitores interessados em mais detalhes sobre análise de estabilidade, principalmente considerando sistemas sujeitos a retardo no tempo via análise funcional são encorajados a consultarem as referências Hale and Lunel (1993) e Gu et al. (2003).

A obtenção dos resultados propostos se fundamenta em utilizar a teoria de estabilidade de Lyapunov-Krasovskii, que corresponde a extensão do método de Lyapunov para tratar sistemas sujeitos a retardo no tempo. Basicamente, esse método requer a construção de um funcional de LyapunovKrasovskii que seja limitado e que leve em conta não só a evolução temporal do sistema em questão, como também seu histórico temporal.

Neste artigo adota-se o seguinte funcional de LyapunovKrasovskii, quadrático e limitado, selecionado de forma similar como apresentada em Palhares et al. (2005):

\footnotetext{
${ }^{1}$ Devido a natureza das RNAs, como sistemas paralelos distribuídos compostos por unidades de processamento, essas possuem uma grande quantidade de canais de comunicação, que podem possuir diferentes comprimentos, que interligam diferentes unidades de processamento. Portanto, um modelo mais realista para RNAs deve considerar diferentes retardos no tempo para os diferentes canais de comunicação. Entretanto, apesar da classe de RNAs considerada como objeto de estudo deste artigo ser sujeita a um retardo no tempo $(d(t) \leq \tau)$ igual em todos os canais de comunicação, os métodos obtidos aqui podem ser facilmente estendidos a RNAs sujeitas a múltiplos retardos no tempo $\left(d_{i}(t) \leq \tau_{i}\right.$ para $\left.i=1, \ldots, n\right)$.
}

$$
V\left(x_{t}\right)=V_{1}\left(x_{t}\right)+V_{2}\left(x_{t}\right)+V_{3}\left(x_{t}\right)
$$

com

$$
\begin{gathered}
V_{1}\left(x_{t}\right) \triangleq e^{2 \alpha t} x^{T}(t) P x(t) \\
V_{2}\left(x_{t}\right) \triangleq \int_{t-d(t)}^{t} e^{2 \alpha s} x^{T}(s) Q x(s) d s \\
V_{3}\left(x_{t}\right) \triangleq \int_{-\tau}^{0} \int_{t+\theta}^{t} e^{2 \alpha s} \dot{x}^{T}(s) Z \dot{x}(s) d s d \theta
\end{gathered}
$$

sendo $P=P^{T}, Q=Q^{T}, Z=Z^{T}$ e $\alpha \geq 0$ um escalar. $\mathrm{O}$ escalar $\alpha$, define se esse funcional será utilizado para obter métodos de estabilidade assintótica ou exponencial, para $\alpha=$ 0 estabilidade assintótica e $\alpha>0$ estabilidade exponencial.

No funcional (10), o objetivo em considerar o termo $V_{2}\left(x_{t}\right)$ é obter um método que leve em conta um limitante superior para taxa de variação do retardo no tempo, $\mu$ em (5). Entretanto, explicitar na formulação do problema apenas o limitante $\mu$, não é suficiente para se obter um método dependente do retardo no tempo. Assim, o motivo de considerar também o termo $V_{3}\left(x_{t}\right)$ é justificado, pois com esse termo é possível explicitar na formulação do problema o limitante superior do retardo no tempo, $\tau$ em (5).

No entanto, neste artigo é considerada uma forma alternativa para atender as condições impostas pela teoria de LyapunovKrasovskii ${ }^{2}$, pois é considerado um funcional quadrático e limitado. Nesse caso, as condições impostas pela teoria de Lyapunov-Krasovskii podem ser reformuladas em uma forma restritiva, como apresentado no próximo Teorema.

Teorema 1 Gu et al. (2003, p. 150). Um sistema sujeito a retardo no tempo é assintoticamente estável se existir um funcional de Lyapunov-Krasovskii quadrático e limitado $V\left(x_{t}\right)$, tal que para algum $\epsilon>0$, satisfaz-se

$$
V\left(x_{t}\right) \geq \epsilon\|x(t)\|^{2}
$$

e sua derivada ao longo das trajetória do sistema satisfaz

$$
\dot{V}\left(x_{t}\right) \leq-\epsilon\|x(t)\|^{2} .
$$

Sendo que, $x_{t}$ corresponde ao valor de $x(t)$ no intervalo $[t-\tau, t]$, para $\tau>0$.

Demonstração: Veja Gu et al. (2003, p. 150).

Tendo, como objetivo métodos de análise de estabilidade formulados em termos de LMIs, que sejam pouco conservadores. Uma técnica considerada anteriormente em $\mathrm{Wu}$ et al.

\footnotetext{
${ }^{2} \mathrm{O}$ teorema de estabilidade de Lyapunov-Krasovskii pode ser encontrado em Gu et al. (2003, p. 12).
} 
(2004), também é utilizada aqui. Nessa técnica, a relação entre o estado no instante $t$ e o estado atrasado, assim como a dependência explicita do retardo no tempo podem ser introduzidas na análise da estabilidade, considerando a fórmula de Leibniz-Newton e matrizes de relaxação. Esta estratégia é detalhada no próximo Lema, que não é explicitamente apresentado na forma de Lema em Wu et al. (2004).

Lema 1 Para quaisquer matrizes $Y$ e $T$ com dimensões apropriadas, o termo nulo

$$
\begin{aligned}
& 2\left[x^{T}(t) Y+x^{T}(t-d(t)) T\right] \times \\
& \quad\left[x(t)-\int_{t-d(t)}^{t} \dot{x}(s) d s-x(t-d(t))\right]=0,
\end{aligned}
$$

é equivalente a

$$
\xi^{T}(t) \Gamma \xi(t)-\int_{t-d(t)}^{t} \zeta^{T}(t) \Upsilon \zeta(t) d s=0,
$$

com

$$
\begin{gathered}
\xi(t) \triangleq\left[\begin{array}{c}
x(t) \\
x(t-d(t))
\end{array}\right], \zeta(t, s) \triangleq\left[\begin{array}{c}
x(t) \\
x(t-d(t)) \\
\dot{x}(s)
\end{array}\right], \\
\Gamma \triangleq\left[\begin{array}{ll}
\Gamma_{11} & \Gamma_{12} \\
\Gamma_{12}^{T} & \Gamma_{22}
\end{array}\right]=\left[\begin{array}{ccc}
Y+Y^{T} & -Y+T^{T} \\
-Y^{T}+T & -T-T^{T}
\end{array}\right] \\
e \Upsilon \triangleq\left[\begin{array}{ccc}
0 & 0 & -Y \\
0 & 0 & -T \\
-Y^{T} & -T^{T} & 0
\end{array}\right] .
\end{gathered}
$$

O Lema a seguir, é utilizado para que os resultados propostos possam ser formulados em termos de LMIs.

Lema 2 - Wu et al. (2004) - Para qualquer matriz semidefinida positiva $X=\left[\begin{array}{ll}X_{11} & X_{12} \\ X_{12}^{T} & X_{22}\end{array}\right] \geq 0$, a seguinte desigualdade se mantém:

$$
\begin{gathered}
\tau \xi^{T}(t) X \xi(t)-\int_{t-d(t)}^{t} \xi^{T}(t) X \xi(t) d s \geq 0, \\
\operatorname{com} \xi(t)=\left[\begin{array}{ll}
x^{T}(t) & x^{T}(t-d(t))
\end{array}\right]^{T} .
\end{gathered}
$$

Finalmente, a definição a seguir apresenta a propriedade de estabilidade exponencial.

Definição 1 Considere os escalares $\alpha>0$ e $\varrho(\alpha)>0$, tais que:

$$
\|x(t)\| \leq \varrho(\alpha) e^{-\alpha t}\left(\sup _{-\tau \leq \theta \leq 0}\|x(\theta)\|\right), \forall t>0
$$

o sistema (8) é exponencialmente estável, sendo $\alpha$ chamado de grau de estabilidade exponencial.

\section{RESULTADOS PRINCIPAIS}

Nesta seção, são propostos métodos de analise das estabilidades assintótica e exponencial para RNAs descritas como em (8) sujeitas a retardo variante no tempo e/ou a incertezas paramétricas do tipo politópicas. Um dos passos principais é adicionar o termo nulo apresentado no Lema 1 na derivada do funcional de Lyapunov-Krasovskii, para se obter condições dependentes do retardo. O primeiro resultado, i.e., estabilidade assintótica, é apresentado no teorema a seguir, o qual pode ser facilmente verificado por meio de ferramentas computacionais baseadas em LMIs.

Teorema 2 Dados os escalares $\tau>0$ e $\mu<1$, limitantes para o valor do retardo no tempo e sua taxa de variação, respectivamente, e suponha que a hipótese $(H)$ seja satisfeita. O sistema (8) é assintoticamente estável se existirem matrizes simétricas definidas positivas $P, Q$ e $Z, X=$ $\left[\begin{array}{ll}X_{11} & X_{12} \\ X_{12}^{T} & X_{22}\end{array}\right] \geq 0$, e quaisquer matrizes $Y$ e $T$ de dimensões apropriadas, tais que as LMIs abaixo sejam satisfeitas:

$$
\Xi=\left[\begin{array}{ccc}
\phi_{11}+Y+Y^{T} & \phi_{12}-Y+T^{T} & \tau\left(-A+\Sigma_{\sigma} W_{0}^{T}\right) Z \\
\phi_{12}^{T}-Y^{T}+T & \phi_{22}-T-T^{T} & \tau \Sigma_{\sigma} W_{1}^{T} Z \\
\tau Z\left(-A+W_{0} \Sigma_{\sigma}\right) & \tau Z W_{1} \Sigma_{\sigma} & -\tau Z
\end{array}\right],
$$$$
\Xi<0 \quad(17)
$$

$\mathrm{e}$

$$
\Psi=\left[\begin{array}{ccc}
X_{11} & X_{12} & -Y \\
X_{12}^{T} & X_{22} & -T \\
-Y^{T} & -T^{T} & Z
\end{array}\right] \geq 0
$$

$\operatorname{com} \phi_{11}=\left(-A+\Sigma_{\sigma} W_{0}^{T}\right) P+P\left(-A+W_{0} \Sigma_{\sigma}\right)+Q+\tau X_{11}$, $\phi_{12}=P W_{1} \Sigma_{\sigma}+\tau X_{12}, \phi_{22}=-(1-\mu) Q+\tau X_{22}$ e $\Sigma_{\sigma}=$ $\operatorname{diag}\left(\sigma_{i}\right)$, para $i, \ldots, n$, sendo $\sigma_{i}$ limitantes superiores em (9).

Demonstração: Veja apêndice, seção A.1.

O segundo resultado obtido, diz respeito a um método de análise de estabilidade exponencial para RNAs sujeitas a retardo no tempo. Sendo este, apresentado a seguir.

Teorema 3 Dados os escalares $\tau>0$ e $\mu<1$, limitantes para o tamanho do retardo no tempo e sua taxa de variação, respectivamente, $\alpha>0$, grau de estabilidade exponencial e suponha que a hipótese $(H)$ seja satisfeita. O sistema (8) é exponencialmente estável se existirem matrizes simétricas definidas positivas $P, Q$ e $Z, X=\left[\begin{array}{ll}X_{11} & X_{12} \\ X_{12}^{T} & X_{22}\end{array}\right] \geq 0$ e matrizes com dimensões apropriadas, $Y$ e $T$ tais que a LMI 
em (18) e a LMI abaixo sejam verificadas:

$$
\bar{\Xi}=\left[\begin{array}{ccc}
\hat{\phi}_{11}+Y+Y^{T} & \hat{\phi}_{12}-Y+T^{T} & \tau\left(-A+\Sigma_{\sigma} W_{0}^{T}\right) Z \\
\hat{\phi}_{12}^{T}-Y^{T}+T & \hat{\phi}_{22}-T-T^{T} & \tau \Sigma_{\sigma} W_{1}^{T} Z \\
\tau Z\left(-A+W_{0} \Sigma_{\sigma}\right) & \tau Z W_{1} \Sigma_{\sigma} & -\tau Z
\end{array}\right]
$$

$\operatorname{com} \hat{\phi}_{11}=2 \alpha P+\left(-A+\Sigma_{\sigma} W_{0}^{T}\right) P+P\left(-A+W_{0} \Sigma_{\sigma}\right)+Q+\tau X_{11}$, $\hat{\phi}_{12}=P W_{1} \Sigma_{\sigma}+\tau X_{12}, \hat{\phi}_{22}=-e^{-2 \alpha \tau}(1-\mu) Q$

$+\tau X_{22}$ e $\Sigma_{\sigma}=\operatorname{diag}\left(\sigma_{i}\right)$, para $i, \ldots, n$, sendo $\sigma_{i}$ limitantes superiores em (9).

Demonstração: Veja apêndice, seção A.2.

Por fim, sendo a última contribuição deste artigo, é mostrado como os Teoremas 2 e 3 podem ser estendidos para o caso de análise de estabilidade robusta quando as matrizes do sistema não são exatamente conhecidas.

Neste caso, é considerado que as incertezas do modelo pertencem a um domínio politópico. Suponha que as matrizes do sistema (8) são reunidas na matriz:

$$
\mathcal{S} \triangleq\left[\begin{array}{lll}
A & W_{0} & W_{1}
\end{array}\right] .
$$

Então, as matrizes do sistema incerto em (8) pertencem a um domínio politópico $\mathcal{P}$ :

$$
\begin{aligned}
\mathcal{P} \triangleq\{\mathcal{S}(\gamma) & \in \mathbb{R}^{n \times 3 n}: \mathcal{S}(\gamma)=\sum_{i=1}^{N} \gamma_{i} \mathcal{S}_{i} \\
& \left.\gamma_{i} \geq 0, i=1, \ldots N ; \sum_{i=1}^{N} \gamma_{i}=1\right\},
\end{aligned}
$$

sendo que $\mathcal{S}_{i}, i=1, \ldots, N$, denotam os vértices do politopo.

Então, por meio do conceito de estabilidade quadrática, amplamente utilizado na literatura, que garante a estabilidade do sistema para todo domínio de incerteza independente de qualquer tipo de variação dos parâmetros incertos. Esse método é fundamentado em garantir a estabilidade do sistema em todo domínio de incerteza baseado na escolha de um funcional de Lyapunov-Krasovskii fixo.

Contudo, pode-se apresentar o teorema a seguir que estabelece condições suficientes para estabilidade robusta de RNAs com retardo no tempo e incertezas no modelo, extensão do Teorema 2 para sistemas incertos.

Teorema 4 Considere o sistema incerto (8) com $S(\gamma) \in \mathcal{P}$. Dados os escalares $\tau>0$ e $\mu<1$, limitantes para os valores dos retardos no tempo e suas taxas de variações, respectivamente, e suponha que a hipótese $(H)$ seja satisfeita. O sistema incerto é robustamente estável se existirem matrizes simétricas definidas positivas $P, Q$ e $Z$, matriz simétrica semi-definida positiva $X$ em (2), e quaisquer matrizes $Y$ e $T$ de dimensões apropriadas, tais que as LMIs (17) e (18) sejam satisfeitas, $\forall i=1, \ldots, N$, com $A, W_{0}, W_{1}$ tendo o índice subscrito $i, i=1, \ldots, N$.

De maneira similar, o Teorema 3 pode ser estendidos para tratar sistemas incertos sujeitos a retardo no tempo. Simplesmente verificando as condições LMIs no Teorema 3, para todos os $N$ vertices do politopo $\mathcal{P}$.

\section{ESTUDO DE CASOS}

Nesta seção, três exemplos numéricos são estudados a fim de evidenciar as potencialidades dos métodos propostos.

Exemplo 1 Babcock and Westervelt (1987) estudaram uma RNA de dois neurônios com dois retardos no tempo:

$$
\left\{\begin{array}{l}
\frac{d x_{1}}{d t}=-x_{1}(t)+a_{1} \tanh \left[x_{2}\left(t-\tau_{1}\right)\right] \\
\frac{d x_{2}}{d t}=-x_{2}(t)+a_{2} \tanh \left[x_{1}\left(t-\tau_{2}\right)\right]
\end{array}\right.
$$

sendo que $a_{1}, a_{2}, \tau_{1}$ e $\tau_{2}$ são constantes positivas.

Em Babcock and Westervelt (1987) foi mostrado que quando $a_{1} a_{2}<-1$ e $\tau_{1}+\tau_{2}$ é limitado por um determinado valor, a origem do sistema (22) é estável. Quando a soma dos atrasos se incrementa até um valor crítico, a origem se torna instável e a rede oscila em um ciclo limite.

Wei and Ruan (1999) confirmaram a análise apresentada em Babcock and Westervelt (1987) e mostraram que a RNA é estável considerando, e.g., $a_{1}=2, a_{2}=-1.5$ e $\tau_{1}+\tau_{2}<$ 0.8 .

Considerando um caso ligeiramente modificado com $\tau_{1}$ e $\tau_{2}$ variantes no tempo, i.e $\tau_{1}=d_{1}(t)$ e $\tau_{2}=d_{2}(t)$. E além disso, impondo $d_{1}(t)=d_{2}(t) \leq \tau$ e $\dot{d}_{1}(t)=\dot{d}_{2}(t) \leq \mu$. Então, escolhendo $a_{1}=2$ e $a_{2}=-1.5$, pode-se obter o maior retardo no tempo $\tau$, considerando a rede neural (8), com as seguintes matrizes,

$$
\begin{gathered}
A=\left[\begin{array}{ll}
1 & 0 \\
0 & 1
\end{array}\right], W_{0}=0, \\
W_{1}=\left[\begin{array}{cc}
0 & 2 \\
-1.5 & 0
\end{array}\right], \quad \Sigma_{\sigma}=\left[\begin{array}{ll}
1 & 0 \\
0 & 1
\end{array}\right] .
\end{gathered}
$$

Aplicando o Teorema 2 obtém-se os resultados apresentados na Tabela 1 . Note que para $d_{1}(t)=d_{2}(t)=\tau$, i.e., com taxa de variação $\mu=0$, obtém-se o retardo no tempo máximo $\tau=0.4283$. A Tabela 1 apresenta também os resultados para o retardo máximo, se eventualmente, o retardo for variante no tempo.

A figura 1 ilustra quando a bifurcação de Hopf ocorre. Note que é muito próximo ao valor encontrado para o retardo no 
Tabela 1: Máximos retardos no tempo permissíveis (Exemplo 1).

\begin{tabular}{c|c|c|c}
\hline$\mu$ & 0 & 0.5 & 0.9 \\
\hline$\tau$ & 0.4283 & 0.2891 & 0.2886 \\
\hline
\end{tabular}
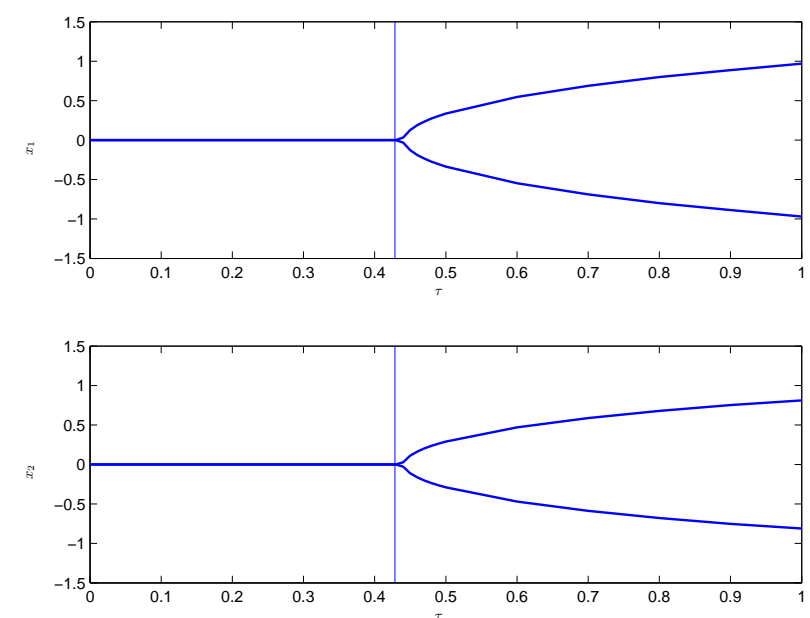

Figura 1: Diagrama de bifurcação para $x_{1}$ e $x_{2}$, respectivamente, fazendo $\tau$ como parâmetro de controle, $\operatorname{com} \mu=0$. A linha vertical marca $\tau=0.4283$.

tempo máximo admitido, i.e., $\tau=0.4283$, o que sugere que o método apresentado é bastante eficiente.

Exemplo 2 Este exemplo trata da análise da estabilidade robusta para uma rede neural (8) sujeita a incertezas paramétricas com 64 vértices, que pode ser representada pelas matrizes:

$$
\begin{array}{r}
A^{(1)}=\left[\begin{array}{cc}
2 & 0 \\
0 & 2.87
\end{array}\right], A^{(2)}=\left[\begin{array}{cc}
4 & 0 \\
0 & 2.87
\end{array}\right], \\
A^{(3)}=\left[\begin{array}{cc}
2 & 0 \\
0 & 2.93
\end{array}\right], A^{(4)}=\left[\begin{array}{cc}
4 & 0 \\
0 & 2.93
\end{array}\right], \\
W_{0}^{(1)}=\left[\begin{array}{cc}
0.4 & 0 \\
0 & 1.98
\end{array}\right], W_{0}^{(2)}=\left[\begin{array}{cc}
1.6 & 0 \\
0 & 1.98
\end{array}\right], \\
W_{0}^{(3)}=\left[\begin{array}{cc}
0.4 & 0 \\
0 & 2.2
\end{array}\right], W_{0}^{(4)}=\left[\begin{array}{cc}
1.6 & 0 \\
0 & 2.2
\end{array}\right], \\
W_{1}^{(1)}=-\left[\begin{array}{cc}
1.1 & 0 \\
1 & 1.3
\end{array}\right], W_{1}^{(2)}=-\left[\begin{array}{cc}
0.9 & 0 \\
1 & 1.3
\end{array}\right], \\
W_{1}^{(3)}=-\left[\begin{array}{cc}
1.1 & 0 \\
1 & 0.7
\end{array}\right], W_{1}^{(4)}=-\left[\begin{array}{cc}
0.9 & 0 \\
1 & 0.7
\end{array}\right], \\
\text { e } \Sigma_{\sigma}=\left[\begin{array}{cc}
1 & 0 \\
0 & 1
\end{array}\right] .
\end{array}
$$

Aplicando o Teorema 4 para a análise da estabilidade robusta obtém-se o retardo no tempo máximo, como mostrado na

\section{Tabela 2.}

Tabela 2: Máximos retardos no tempo obtidos para o Exemplo 2 .

\begin{tabular}{c|c|c|c}
\hline$\mu$ & 0 & 0.5 & 0.9 \\
\hline$\tau$ & 1.0152 & 0.8820 & 0.7799 \\
\hline
\end{tabular}

Exemplo 3 Este exemplo considera a mesma RNA estudada no exemplo 1. Aplicando o Teorema 3 obtém-se os resultados apresentados nas Tabelas 3, 4 e 5 que mostram os graus de decaimento exponencial, $\alpha$, para um determinado limitante superior para o retardo no tempo $\tau$. Sendo que, as Tabelas 3, 4 e 5 foram obtidas para a taxa de variação do retardo $\mu=0, \mu=0.5$ e $\mu=0.9$, respectivamente.

Nas figuras a seguir, Figuras 2-5, são apresentadas as respostas temporais da rede para os decaimentos exponenciais apresentados na Tabela 3 .

Tabela 3: Graus de estabilidade exponencial, $\alpha$, para os retardos no tempo com $\mu=0$ (Exemplo 3).

\begin{tabular}{c|c|c|c|c|c}
\hline$\tau$ & 0.1 & 0.2 & 0.3 & 0.4 & 0.4283 \\
\hline$\alpha$ & 0.68 & 0.37 & 0.16 & 0.02 & 0.00006 \\
\hline
\end{tabular}

Tabela 4: Graus de estabilidade exponencial, $\alpha$, para os retardos no tempo com $\mu=0.5$ (Exemplo 3).

\begin{tabular}{c|c|c|c}
\hline$\tau$ & 0.1 & 0.2 & 0.2891 \\
\hline$\alpha$ & 0.67 & 0.34 & 0.0001 \\
\hline
\end{tabular}

Tabela 5: Graus de estabilidade exponencial, $\alpha$, para os retardos no tempo com $\mu=0.9$ (Exemplo 3).

\begin{tabular}{c|c|c|c}
\hline$\tau$ & 0.1 & 0.2 & 0.2886 \\
\hline$\alpha$ & 0.67 & 0.34 & 0.0003 \\
\hline
\end{tabular}

\section{AGRADECIMENTOS}

Este trabalho recebeu o apoio das agências CNPq, CAPES e FAPEMIG.

\section{CONCLUSÃo}

Neste artigo discutiu-se o problema de estabilidade assintótica e exponencial em uma classe de RNAs (Redes Neurais 

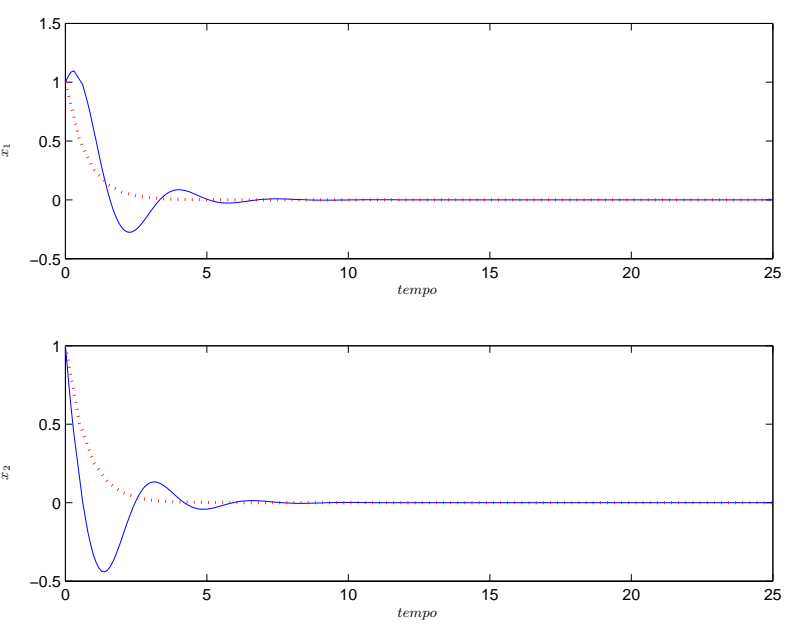

Figura 2: Resposta temporal da RNA com $\tau=0.1$ (traço contínuo) e decaimento exponencial com $\alpha=0.68$ (pontilhado) para $\mu=0$.
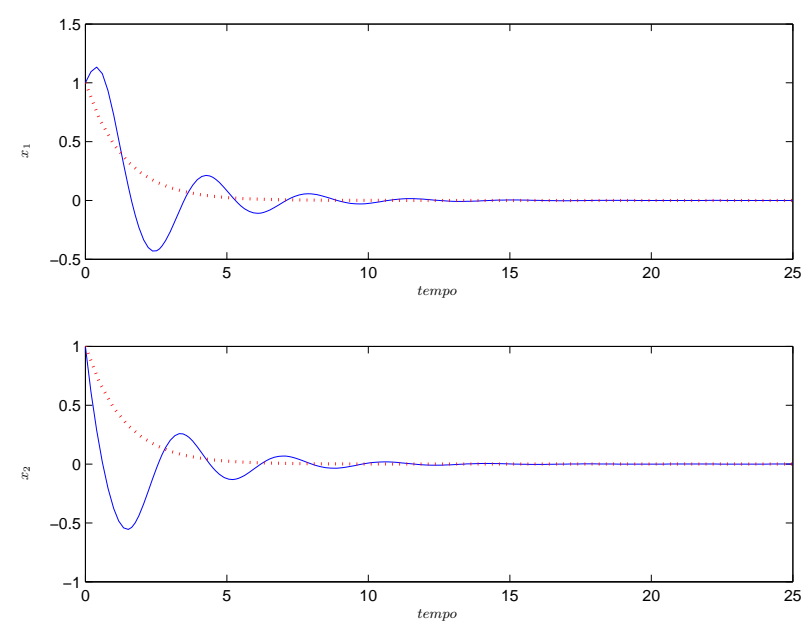

Figura 3: Resposta temporal da RNA com $\tau=0.2$ (traço contínuo) e decaimento exponencial com $\alpha=0.37$ (pontilhado) para $\mu=0$.

Artificiais) sujeitas a influência de retardo no tempo (constante ou variante) e/ou incertezas paramétricas do tipo politópicas. Condições suficientes, dependentes do retardo no tempo, foram obtidas em termos de LMIs.

Os resultados obtidos nos exemplos sugerem que os métodos propostos são eficientes e que é possível encontrar uma boa aproximação para o máximo retardo no tempo que garante a estabilidade assintótica, além do grau de decaimento exponencial da solução, o qual pode ser utilizado como uma estimativa do tempo de acomodação de RNAs.

Em questões práticas, os métodos desenvolvidos poderão ser
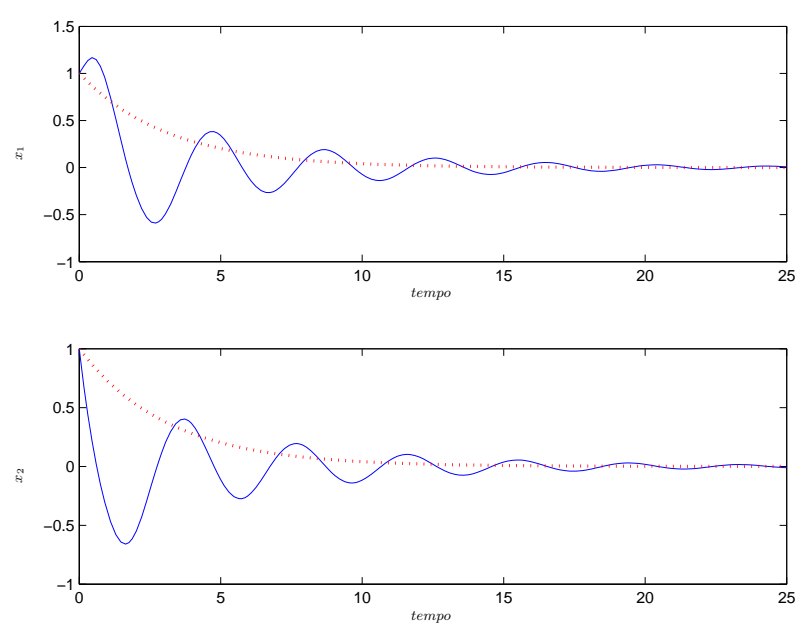

Figura 4: Resposta temporal da RNA com $\tau=0.3$ (traço contínuo) e decaimento exponencial com $\alpha=0.16$ (pontilhado) para $\mu=0$.
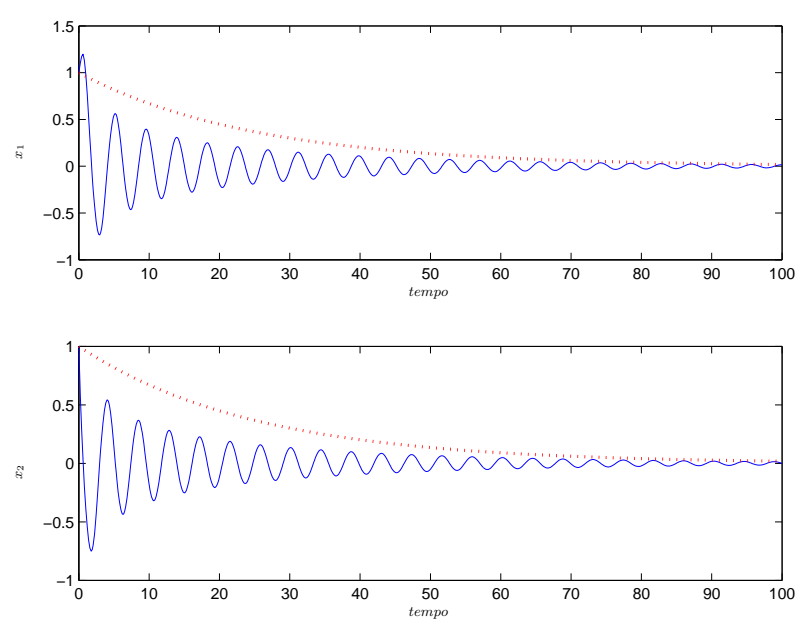

Figura 5: Resposta temporal da RNA com $\tau=0.4$ (traço contínuo) e decaimento exponencial com $\alpha=0.02$ (pontilhado) para $\mu=0$.

aplicados para avaliar a influência do retardo no tempo e/ou de incertezas paramétricas em uma classe de RNAs implementadas analogicamente, ou considerando um quesito de projeto, os métodos propostos poderão auxiliar um projetista a escolher uma determinada classe de amplificadores operacionais, ou até mesmo, a implementar RNAs com parâmetros ajustáveis.

\section{A APÊNDICE}

Neste apêndice são apresentadas as demonstrações dos teoremas propostos. 


\section{A.1 Demonstração do Teorema 2}

Demonstração: Considere o funcional do tipo LyapunovKrasovskii em (10) com $\alpha=0, P>0, Q>0$ e $Z>0$, então a condição (11) no Teorema 1 é atendida.

Agora, derivando o funcional em (10) $\operatorname{com} \alpha=0$, em relação as trajetórias da RNA em (8), obtém-se:

$$
\dot{V}\left(x_{t}\right)=\dot{V}_{1}\left(x_{t}\right)+\dot{V}_{2}\left(x_{t}\right)+\dot{V}_{3}\left(x_{t}\right),
$$

com

$$
\begin{gathered}
\dot{V}_{1}\left(x_{t}\right)=x^{T}(t) P \dot{x}(t)+\dot{x}^{T}(t) P x(t) \\
=x^{T}(t) P\left[-A x(t)+W_{0} f(x(t))+W_{1} f(x(t-d(t)))\right] \\
+\left[-A x(t)+W_{0} f(x(t))+W_{1} f(x(t-d(t)))\right]^{T} P x(t) \\
=x^{T}(t) P\left[-A x(t)+W_{0} f(x(t))+W_{1} f(x(t-d(t)))\right] \\
+\left[-x^{T}(t) A+f^{T}(x(t)) W_{0}^{T}+f^{T}(x(t-d(t))) W_{1}^{T}\right] P x(t) \\
=-x^{T}(t)[P A+A P] x(t)+f^{T}(x(t)) W_{0}^{T} P x(t) \\
+x^{T}(t) P W_{0} f(x(t))+2 x^{T}(t) P W_{1} f(x(t-d(t))) \\
\leq-x^{T}(t)[P A+A P] x(t)+x^{T}(t) \Sigma_{\sigma} W_{0}^{T} P x(t) \\
+x^{T}(t) P W_{0} \Sigma_{\sigma} x(t)+2 x^{T}(t) P W_{1} \Sigma_{\sigma} x(t-d(t)) \\
=x^{T}(t)\left[-P A-A P+\Sigma_{\sigma} W_{0}^{T} P+P W_{0} \Sigma_{\sigma}\right] x(t) \\
\quad+2 x^{T}(t)\left(P W_{1} \Sigma_{\sigma} x(t-d(t)),\right.
\end{gathered}
$$

$$
\begin{aligned}
& \dot{V}_{2}\left(x_{t}\right)=-(1-\dot{d}(t)) x^{T}(t-d(t)) Q x(t-d(t))+x^{T}(t) Q x(t) \\
& \leq-(1-\mu) x^{T}(t-d(t)) Q x(t-d(t))+x^{T}(t) Q x(t),
\end{aligned}
$$

$$
\begin{gathered}
\dot{V}_{3}\left(x_{t}\right)=\tau \dot{x}^{T}(t) Z \dot{x}(t)-\int_{t-\tau}^{t} \dot{x}^{T}(s) Z \dot{x}(s) d s \\
=\tau\left[-A x(t)+W_{0} f(x(t))+W_{1} f(x(t-d(t)))\right]^{T} \\
\times Z\left[-A x(t)+W_{0} f(x(t))+W_{1} f(x(t-d(t)))\right] \\
-\int_{t-\tau}^{t} \dot{x}^{T}(s) Z \dot{x}(s) d s \\
=\tau\left[-x^{T}(t) A+f^{T}(x(t)) W_{0}^{T}+f^{T}(x(t-d(t))) W_{1}^{T}\right] \\
\times Z\left[-A x(t)+W_{0} f(x(t))+W_{1} f(x(t-d(t)))\right] \\
\quad-\int_{t-\tau}^{t} \dot{x}^{T}(s) Z \dot{x}(s) d s \\
\leq \tau\left[-x^{T}(t) A+x^{T}(t) \Sigma_{\sigma} W_{0}^{T}+x^{T}(t-d(t)) \Sigma_{\sigma} W_{1}^{T}\right] \\
\times Z\left[-A x(t)+W_{0} \Sigma_{\sigma} x(t)+W_{1} \Sigma_{\sigma} x(t-d(t))\right] \\
\quad-\int_{t-\tau}^{t} \dot{x}^{T}(s) Z \dot{x}(s) d s
\end{gathered}
$$

$\operatorname{com} \xi(t)$ definido no Lema $1 \mathrm{e}$

$$
\begin{aligned}
\Omega= & {\left[\begin{array}{ll}
\Omega_{11} & \Omega_{12} \\
\Omega_{12}^{T} & \Omega_{22}
\end{array}\right], } \\
& \left\{\begin{array}{l}
\Omega_{11}=\tau\left(-A+\Sigma_{\sigma} W_{0}^{T}\right) Z\left(-A+W_{0} \Sigma_{\sigma}\right), \\
\Omega_{12}=\tau\left(-A+\Sigma_{\sigma} W_{0}^{T}\right) Z\left(W_{1} \Sigma_{\sigma}\right), \\
\Omega_{22}=\tau\left(\Sigma_{\sigma} W_{1}^{T}\right) Z\left(W_{1} \Sigma_{\sigma}\right) .
\end{array}\right.
\end{aligned}
$$

Adicionando em (23) o termo nulo apresentado no Lema 1, e qualquer matriz semi-definida positiva como no Lema 2, obtém-se:

$$
\begin{aligned}
\dot{V}\left(x_{t}\right) \leq & \dot{V}_{1}\left(x_{t}\right)+\dot{V}_{2}\left(x_{t}\right)+\dot{V}_{3}\left(x_{t}\right) \\
& +2\left[x^{T}(t) Y+x^{T}(t-d(t)) T\right] \\
& \times\left[x(t)-\int_{t-d(t)}^{t} \dot{x}(s) d s-x(t-d(t))\right] \\
& +\tau \xi^{T}(t) X \xi(t)-\int_{t-d(t)}^{t} \xi^{T}(t) X \xi(t) d s .
\end{aligned}
$$

Substituindo $\dot{V}_{1}\left(x_{t}\right), \dot{V}_{2}\left(x_{t}\right)$ e $\dot{V}_{3}\left(x_{t}\right)$ em (28) por seus limitantes dados em (24), (25) e (26), respectivamente, resulta 
em:

$$
\begin{aligned}
& \dot{V}\left(x_{t}\right) \leq x^{T}\left[-P A-A P+\Sigma_{\sigma} W_{0}^{T} P+P W_{0} \Sigma_{\sigma}\right] x(t) \\
&+2 x^{T}(t) P W_{1} \Sigma_{\sigma} x(t-d(t)) \\
&-(1-\mu) x^{T}(t-d(t)) Q x(t-d(t))+x^{T}(t) Q x(t)+\xi^{T}(t) \Omega \xi(t)-\int_{t-\tau}^{t} \dot{x}^{T}(s) Z \dot{x}(s) d s \\
& \quad+2\left[x^{T}(t) Y+x^{T}(t-d(t)) T\right] \\
& \times\left[x(t)-\int_{t-d(t)}^{t} \dot{x}(s) d s-x(t-d(t))\right] \\
&+\tau \xi^{T}(t) X \xi(t)-\int_{t-d(t)}^{t} \xi^{T}(t) X \xi(t) d s .
\end{aligned}
$$

Logo, reescrevendo a desigualdade anterior na forma quadrática, obtém-se:

$$
\dot{V}\left(x_{t}\right) \leq \xi^{T}(t) \tilde{\Xi} \xi(t)-\int_{t-d(t)}^{t} \zeta^{T}(t, s) \Psi \zeta(t, s) d s .
$$

sendo $\zeta(t, s)$ e $\Psi$ definidos no Lema 1 e

$$
\tilde{\Xi}=\left[\begin{array}{cc}
\phi_{11}+\Omega_{11}+\Gamma_{11} & \phi_{12}+\Omega_{12}+\Gamma_{12} \\
\phi_{12}^{T}+\Omega_{12}^{T}+\Gamma_{12}^{T} & \phi_{22}+\Omega_{22}+\Gamma_{22}
\end{array}\right]
$$

$\operatorname{com} \phi_{11}=-P A-A P+\Sigma_{\sigma} W_{0}^{T} P+P W_{0} \Sigma_{\sigma}+Q+\tau X_{11}$, $\phi_{12}=P W_{1} \Sigma_{\sigma}+\tau X_{12}, \phi_{22}=-(1-\mu) Q+\tau X_{22} . \Gamma_{11}, \Gamma_{12}$ e $\Gamma_{22}$ são definidos em (14) e $\Omega_{11}, \Omega_{12}, \Omega_{22}$ são definidos em (27).

Se $\tilde{\Xi}<0, \Psi \geq 0$, garante que a condição (12) no Teorema 1 seja atendida. Finalmente, aplicando o complemento de Schur em $\tilde{\Xi}$ obtém-se $\Xi$ em (17). Portanto, a origem do sistema (8) é assintoticamente estável se as LMIs em definidas no Teorema 2 forem satisfeitas.

\section{A.2 Demonstração do Teorema 3}

O seguinte lema é utilizado na seguinte demonstração.

Lema 3 Para quaisquer vetores $a, b \in \mathbb{R}^{n}$, a seguinte desigualdade

$$
2 a^{T} b \leq a^{T} X a+b^{T} X^{-1} b,
$$

é assegurada, se somente se $X>0$.

Demonstração: Considere o funcional do tipo LyapunovKrasovskii em (10) com $\alpha>0, P>0, Q>0$ e $Z>0$, então a condição (11) no Teorema 1 é atendida.
Agora derivando o funcional em (10) $\operatorname{com} \alpha>0$, em relação as trajetórias da RNA em (8), obtém-se:

$$
\dot{V}\left(x_{t}\right)=\dot{V}_{1}\left(x_{t}\right)+\dot{V}_{2}\left(x_{t}\right)+\dot{V}_{3}\left(x_{t}\right) .
$$

com

$$
\begin{gathered}
\dot{V}_{1}\left(x_{t}\right)=2 \alpha e^{2 \alpha t} x^{T}(t) P x(t) \\
+e^{2 \alpha t} \dot{x}^{T}(t) P x(t)+e^{2 \alpha t} x^{T}(t) P \dot{x}(t) \\
=e^{2 \alpha t}\left[x^{T}(t) 2 \alpha P x(t)+\dot{x}^{T}(t) P x(t)+x^{T}(t) P \dot{x}(t)\right] \\
=e^{2 \alpha t}\left[x^{T}(t) 2 \alpha P x(t)\right. \\
+\left[-x^{T}(t) A+f^{T}(x(t)) W_{0}^{T}+f^{T}(x(t-d(t))) W_{1}^{T}\right] P x(t) \\
\left.+x^{T}(t) P\left[-A x(t)+W_{0} f(x(t))+W_{1} f(x(t-d(t)))\right]\right] \\
=e^{2 \alpha t}\left[x^{T}(t) 2 \alpha P x(t)\right. \\
-x^{T}(t) P A x(t)-x^{T}(t) A P x(t) \\
+f^{T}(x(t)) W_{0}^{T} P x(t)+x^{T}(t) P W_{0} f(x(t)) \\
\left.+2 x^{T}(t) P W_{1} f(x(t-d(t)))\right] \\
\leq e^{2 \alpha t} x^{T}(t)[2 \alpha P-P A-A P] x(t) \\
+x^{T}(t) \Sigma_{\sigma} W_{0}^{T} P x(t)+x^{T}(t) P W_{0} \Sigma_{\sigma} x(t) \\
+2 x^{T}(t) P W_{1} \Sigma_{\sigma} x(t-d(t)) \\
=e^{2 \alpha t}\left\{x^{T}(t)\left[2 \alpha P-P A-A P+\Sigma_{\sigma} W_{0}^{T} P+P W_{0} \Sigma_{\sigma}\right] x(t)\right. \\
\left.+2 x^{T}(t) P W_{1} \Sigma_{\sigma} x(t-d(t))\right\}
\end{gathered}
$$

$$
\begin{gathered}
\dot{V}_{2}\left(x_{t}\right)=-e^{2 \alpha(t-d(t))}(1-\dot{d}(t)) x^{T}(t-d(t)) Q x(t-d(t)) \\
+e^{2 \alpha t} x^{T}(t) Q x(t) \\
\leq-e^{2 \alpha(t-\tau)}(1-\dot{d}(t)) x^{T}(t-d(t)) Q x(t-d(t)) \\
+e^{2 \alpha t} x^{T}(t) Q x(t) \\
=-e^{2 \alpha t} e^{-2 \alpha \tau}(1-\dot{d}(t)) x^{T}(t-d(t)) Q x(t-d(t)) \\
+e^{2 \alpha t} x^{T}(t) Q x(t) \\
=e^{2 \alpha t}\left\{-e^{-2 \alpha \tau}(1-\dot{d}) x^{T}(t-d(t)) Q x(t-d(t))\right. \\
\left.\quad+x^{T}(t) Q x(t)\right\} \\
\leq e^{2 \alpha t}\left\{-e^{-2 \alpha \tau}(1-\mu) x^{T}(t-d(t)) Q x(t-d(t))\right. \\
\left.+x^{T}(t) Q x(t)\right\}
\end{gathered}
$$




$$
\begin{gathered}
\dot{V}_{3}\left(x_{t}\right)=\tau e^{2 \alpha t} \dot{x}^{T}(t) Z \dot{x}(t)-\int_{t-\tau}^{t} e^{2 \alpha t} \dot{x}^{T}(s) Z \dot{x}(s) d s \\
=\tau e^{2 \alpha t}\left[-A x(t)+W_{0} f(x(t))+W_{1} f(x(t-d(t)))\right]^{T} \\
\times Z\left[-A x(t)+W_{0} f(x(t))+W_{1} f(x(t-d(t)))\right] \\
\quad-\int_{t-\tau}^{t} e^{2 \alpha t} \dot{x}^{T}(s) Z \dot{x}(s) d s \\
=\tau e^{2 \alpha t}\left[x^{T}(t)\left(-A+\Sigma_{\sigma} W_{0}^{T}\right)+x^{T}(t-d(t)) \Sigma_{\sigma} W_{1}^{T}\right] \\
\quad \times Z\left[\left(-A+W_{0} \Sigma_{\sigma}\right) x(t)+W_{1} \Sigma_{\sigma} x(t-d(t))\right] \\
\quad-\int_{t-\tau}^{t} e^{2 \alpha t} \dot{x}^{T}(s) Z \dot{x}(s) d s \\
\leq e^{2 \alpha t}\left\{\xi^{T}(t) \Omega \xi(t)-\int_{t-d(t)}^{t} \dot{x}^{T}(s) Z \dot{x}(s) d s\right\},
\end{gathered}
$$

sendo que $\xi(t)$ é definido no Lema 1 e $\Omega$ é definido em (27). Substituindo $\dot{V}_{1}\left(x_{t}\right), \dot{V}_{2}\left(x_{t}\right)$ e $\dot{V}_{3}\left(x_{t}\right)$ em (32) por (33), (34) e (35), respectivamente, obtém-se

$$
\begin{gathered}
\dot{V}\left(x_{t}\right) \leq e^{2 \alpha t}\left\{x ^ { T } ( t ) \left[2 \alpha P-P A-A P+\Sigma_{\sigma} W_{0}^{T} P\right.\right. \\
\left.\left.+P W_{0} \Sigma_{\sigma}\right] x(t)+2 x^{T}(t) P W_{1} \Sigma_{\sigma} x(t-d(t))\right\} \\
+e^{2 \alpha t}\left\{-e^{-2 \alpha \tau}(1-\mu) x^{T}(t-d(t)) Q x(t-d(t))+x^{T}(t) Q x(t)\right\} \\
+e^{2 \alpha t}\left\{\xi^{T}(t) \Omega \xi(t)-\int_{t-d(t)}^{t} \dot{x}^{T}(s) Z \dot{x}(s) d s\right\} \\
=e^{2 \alpha t}\left\{x ^ { T } ( t ) \left[2 \alpha P-P A-A P+\Sigma_{\sigma} W_{0}^{T} P\right.\right. \\
\left.+P W_{0} \Sigma_{\sigma}+Q\right] x(t)+2 x^{T}(t) P W_{1} \Sigma_{\sigma} x(t-d(t)) \\
-e^{-2 \alpha \tau}(1-\mu) x^{T}(t-d(t)) Q x(t-d(t)) \\
\left.+\xi^{T}(t) \Omega \xi(t)-\int_{t-d(t)}^{t} \dot{x}^{T}(s) Z \dot{x}(s) d s\right\} \\
\dot{V}\left(x_{t}\right) \leq e^{2 \alpha t} \Pi
\end{gathered}
$$

com

$$
\begin{gathered}
\Pi \triangleq x^{T}(t)\left[2 \alpha P-P A-A P+\Sigma_{\sigma} W_{0}^{T} P+P W_{0} \Sigma_{\sigma}+Q\right] x(t) \\
\quad+2 x^{T}(t) P W_{1} \Sigma_{\sigma} x(t-d(t)) \\
-e^{-2 \alpha \tau}(1-\mu) x^{T}(t-d(t)) Q x(t-d(t)) \\
+\xi^{T}(t) \Omega \xi(t)-\int_{t-d(t)}^{t} \dot{x}^{T}(s) Z \dot{x}(s) d s
\end{gathered}
$$

Adicionando em (37) o termo nulo no Lema 1 e qualquer matriz semi-definida positiva como no Lema 2 , segue que:

$$
\begin{aligned}
\Pi \leq x^{T}(t)\left[2 \alpha P-P A-A P+\Sigma_{\sigma} W_{0}^{T} P+P W_{0} \Sigma_{\sigma}+Q\right] x(t) \\
\quad+2 x^{T}(t) P W_{1} \Sigma_{\sigma} x(t-d(t)) \\
-e^{-2 \alpha \tau}(1-\mu) x^{T}(t-d(t)) Q x(t-d(t)) \\
+\xi^{T}(t) \Omega \xi(t)-\int_{t-d(t)}^{t} \dot{x}^{T}(s) Z \dot{x}(s) d s \\
\quad+2\left[x^{T}(t) Y+x^{T}(t-d(t)) T\right] \\
\times\left[x(t)-\int_{t-d(t)}^{t} \dot{x}(s) d s-x(t-d(t))\right] \\
+\tau \xi^{T}(t) X \xi(t)-\int_{t-d(t)}^{t} \xi^{T}(t) X \xi(t) d s \\
=\xi^{T}(t) \hat{\Xi} \xi(t)-\int_{t-d(t)}^{t} \zeta^{T}(t, s) \Psi \zeta(t, s) d s . \quad \text { (38) }
\end{aligned}
$$

$\operatorname{com} \zeta(t, s)$ e $\Psi$ definidos no Lema $1 \mathrm{e}$

$$
\hat{\Xi}=\left[\begin{array}{ll}
\hat{\phi}_{11}+\Omega_{11}+\Gamma_{11} & \hat{\phi}_{12}+\Omega_{12}+\Gamma_{12} \\
\hat{\phi}_{12}^{T}+\Omega_{12}^{T}+\Gamma_{12}^{T} & \hat{\phi}_{22}+\Omega_{22}+\Gamma_{22}
\end{array}\right]
$$

sendo $\hat{\phi}_{11}=2 \alpha P-P A-A P+\Sigma_{\sigma} W_{0}^{T} P+P W_{0} \Sigma_{\sigma}+Q+$ $\tau X_{11}, \hat{\phi}_{12}=P W_{1} \Sigma_{\sigma}+\tau X_{12}, \hat{\phi}_{22}=-e^{-2 \alpha \tau}(1-\mu) Q+$ $\tau X_{22} . \Gamma_{11}, \Gamma_{12}$ e $\Gamma_{22}$ são definidos em (14) e $\Omega_{11}, \Omega_{12}, \Omega_{22}$ são definidos em (27).

Então substituindo (38) em (36):

$\dot{V}\left(x_{t}\right) \leq e^{2 \alpha t}\left\{\xi^{T}(t) \hat{\Xi} \xi(t)-\int_{t-d(t)}^{t} \zeta^{T}(t, s) \Psi \zeta(t, s) d s\right\}$.

Se $\hat{\Xi}<0, \Psi \geq 0$, garante que a condição (12) no Teorema 1 seja atendida. Note que, aplicando o complemento de Schur em $\hat{\Xi}$ obtém-se $\bar{\Xi}$ em (19).

Conseqüentemente, segue que

$$
V\left(x_{t}\right) \leq V\left(x_{t 0}\right), \quad \forall t \geq 0,
$$

sendo $V\left(x_{t 0}\right)=\left.V\left(x_{t}\right)\right|_{t=0}$. Considerando (10), pode-se notar que

$$
\begin{aligned}
V\left(x_{t}\right) \leq & e^{2 \alpha t} \lambda_{\max }(P)\|x(t)\|^{2} \\
& +\lambda_{\max }(Q) \int_{t-d(t)}^{t} e^{2 \alpha s}\|x(s)\|^{2} d s \\
& +\lambda_{\max }(Z) \int_{-\tau}^{0} \int_{t+\theta}^{t} e^{2 \alpha s}\|\dot{x}(s)\|^{2} d s d \theta .
\end{aligned}
$$


Além disso, note que

$$
\begin{gathered}
\int_{-\tau}^{0} \int_{t+\theta}^{t} e^{2 \alpha s}\|\dot{x}(s)\|^{2} d s d \theta \\
=\int_{t-\tau}^{t} \int_{-\tau}^{s-t} e^{2 \alpha s}\|\dot{x}(s)\|^{2} d \theta d s \\
\leq \tau \int_{t-\tau}^{t} e^{2 \alpha s}\|\dot{x}(s)\|^{2} d s
\end{gathered}
$$

Então, considerando (42), (41) pode ser reescrito da seguinte forma

$$
\begin{aligned}
& V\left(x_{t}\right) \leq e^{2 \alpha t} \lambda_{\max }(P)\|x(t)\|^{2} \\
& \quad+\lambda_{\max }(Q) \int_{t-d(t)}^{t} e^{2 \alpha s}\|x(s)\|^{2} d s \\
& \quad+\tau \lambda_{\max }(Z) \int_{t-\tau}^{t} e^{2 \alpha s}\|\dot{x}(s)\|^{2} d s .
\end{aligned}
$$

Agora, fazendo $t=0$ em (43), utilizando o Lema 3, considerando $(8)$ e a hipótese $(\mathrm{H})$, pode-se deduzir que

$$
\begin{aligned}
& V\left(x_{t 0}\right) \leq \lambda_{\max }(P)\|x(0)\|^{2} \\
&+\lambda_{\max }(Q) \int_{-d(0)}^{0} e^{2 \alpha s}\|x(s)\|^{2} d s \\
&+\tau \lambda_{\max }(Z) \int_{-\tau}^{0} e^{2 \alpha s}\left[\lambda_{\max }\left(-A+W_{0} \Sigma_{\sigma}\right) x(s)\right.\left.+\lambda_{\max }\left(W_{1} \Sigma_{\sigma}\right) x(s-d(s))\right]^{T} \\
& \times\left[\lambda_{\max }(-\right.\left.\left.+W_{0} \Sigma_{\sigma}\right) x(s)+\lambda_{\max }\left(W_{1} \Sigma_{\sigma}\right) x(s-d(s))\right] d s \\
& \leq \lambda_{\max }(P)\|\phi\|^{2}+\lambda_{\max }(Q)\|\phi\|^{2} \int_{-d(0)}^{0} e^{2 \alpha s} d s \\
&+2 \tau \lambda_{\max }(Z)\left[\lambda_{\max }^{2}\left(-A+W_{0} \Sigma_{\sigma}\right)\right. \\
&\left.+\lambda_{\max }^{2}\left(W_{1} \Sigma_{\sigma}\right)\right]\|\phi\|^{2} \int_{-\tau}^{0} e^{2 \alpha s} d s \\
& \leq \lambda_{\max }(P)\|\phi\|^{2}+\lambda_{\max }(Q)\|\phi\|^{2}\left(\frac{1-e^{-2 \alpha \tau}}{2 \alpha}\right) \\
&+2 \tau \lambda_{\max }(Z)\left[\lambda_{\max }^{2}\left(-A+W_{0} \Sigma_{\sigma}\right)\right. \\
&\left.+\lambda_{\max }^{2}\left(W_{1} \Sigma_{\sigma}\right)\right]\|\phi\|^{2}\left(\frac{1-e^{-2 \alpha \tau}}{2 \alpha}\right), \quad(44)
\end{aligned}
$$

sendo que, $\|\phi\| \triangleq \sup _{-\tau \leq \theta \leq 0}\|x(\theta)\|$.

Por outro lado, tem-se

$$
V\left(x_{t}\right) \geq e^{2 \alpha t} x^{T}(t) P x(t) \geq e^{2 \alpha t} \lambda_{\min }(P)\|x(t)\|^{2} .
$$

Portanto, pode-se concluir de $V\left(x_{t}\right) \leq V\left(x_{t 0}\right)$, utilizando (44) e (45), que

$$
\|x(t)\| \leq \varrho(\alpha) e^{-\alpha t}\|\phi\|, \quad \forall t>0
$$

com

$$
\begin{aligned}
& \varrho(\alpha) \triangleq \sqrt{\frac{\eta}{\lambda_{\min }(P)}}, \\
& \eta=\lambda_{\max }(P)+\lambda_{\max }(Q)\left(\frac{1-e^{-2 \alpha \tau}}{2^{\alpha}}\right) \\
& +2 \tau \lambda_{\max }(Z) \lambda_{\max }^{2}\left(-A+W_{0} \Sigma_{\sigma}\right)\left(\frac{1-e^{-2 \alpha \tau}}{2^{\alpha}}\right) \\
& \quad+2 \tau \lambda_{\max }(Z) \lambda_{\max }^{2}\left(W_{1} \Sigma_{\sigma}\right)\left(\frac{1-e^{-2 \alpha \tau}}{2^{\alpha}}\right)
\end{aligned}
$$

Então, da Definição 1, o sistema (8) é exponencialmente estável.

\section{REFERÊNCIAS}

Arik, S. (2000). Stability analysis of delayed neural networks, IEEE Transactions on Circuits and Systems - Part I 47: 381-402.

Babcock, K. L. and Westervelt, R. M. (1987). Dynamics of simple electronic neural networks, Physica D 28: 305316.

Baldi, P. and Atiya, A. F. (1994). How delays affect neural dynamics and learning, IEEE Transactions Neural Networks 5: 612-621.

Cao, J. (2000). Periodic oscillation and exponential stability of delayed CNNs, Physics Letters A 270: 157-163.

Chen, T. and Rong, L. (2003). Delay-independent stability analysis of Cohen-Grossberg neural networks., Physics Letters A 317: 436-449.

Chen, W.-H., Guan, Z.-H. and Lu, X. (2004). Delaydependent exponential stability of neural networks with variable delays, Physics Letters A 326: 355-363.

den Driessche, P. V. and Zou, X. (1998). Global attractivity in delayed Hopfield neural networks model, SIAM Journal on Applied Mathematics 58(6): 1878-1890.

Ensari, T. and Arik, S. (2005). Global stability of a class of neural networks with time-varying delay, IEEE Transactions on Circuits and Systems-II: Express Briefs 52(3): 126-130.

Gopalsamy, K. and He, X. Z. (1994a). Delay-independent stability in bidirectional associative memory networks, IEEE Transactions on Neural Networks 5: 998-1002.

Gopalsamy, K. and He, X. Z. (1994b). Stability in asymmetric Hopfield networks with transmission delays, Physica D 76: 344-358. 
Gu, K., Kharitonov, V. and Chen, J. (2003). Stability of timedelay systems, Birkhäuser, Boston, MA.

Hale, J. K. and Lunel, S. M. V. (1993). Introduction to functional differential equations, Spring-Verlag, New York.

Hopfield, J. J. (1982). Neural networks and physics systems with emergent collective computation abilities, Proceedings of the National Academic Science of the USA 79(2): 2554-2558.

Joy, M. P. (1999). On the global convergence of a class of functional differential equations with applications in neural network theory, Journal of Mathematical Analysis and Applications 232: 61-81.

Joy, M. P. (2000). Results concerning the absolute stability of delayed neural networks, Neural Networks 13: 613616.

Li, C., Liao, X. and Zhang, R. (2004). Global robust asymptotical stability of multi-delayed interval neural networks: an LMI approach, Physics Letters A 328: 452-462.

Liao, X., Chen, G. and Sanchez, E. N. (2002a). Delaydependent exponential stability analysis of delayed neural networks: an LMI approach, Neural Networks 15(7): 855-866.

Liao, X., Chen, G. and Sanchez, E. N. (2002b). LMI-based approach for asymptotically stability analysis of delayed neural networks, IEEE Transactions on Circuits and Systems - Part I 49(3): 1033-1039.

Liao, X. F. and Yu, J. B. (1998). Qualitative analysis of bidirectional associative memory networks with time delays, International Journal of Circuit Theory and Applications 26: 219-229.

Liao, X. and J.Yu (1998). Robust stability for interval Hopfield neural networks with time delay, IEEE Transactions Neural Networks 9: 1042-1046.

Marcus, C. M. and Westervelt, R. M. (1989). Stability of analogy neural networks with delay, Physics Reviews A 39(2): 347-359.

Morita, M. (1993). Associative memory with nonmonotone dynamics, Neural Networks 6: 115-126.

Morita, S. Y. M. and Amari, S. (1993). Capacity of associative memory using a nonmonotonic neuron model, Neural Networks 6(6): 167-176.

Palhares, R. M., Campos, C. D., Ekel, P. Y., Leles, M. C. R. and D'Angelo, M. F. S. V. (2005). Delay-dependent robust $\mathcal{H}_{\infty}$ control of uncertain linear systems with lumped delays, IEE Proceedings Control Theory and Applications 152(1): 27-33.
Roska, T., Wu, C. W., Balsi, M. and Chua, L. O. (1992). Stability and dynamics of delay-type general neural networks, IEEE Transactions on Circuits and Systems - Part I 39: 487-490.

Roska, T., Wu, C. W. and Chua, L. O. (1993). Stability of cellular neural network with dominant nonlinear and delay-type templates, IEEE Transactions on Circuits and Systems - Part I 40: 270-272.

Singh, V. (2004). Robust stability of cellular neural networks with delay: linear matrix inequality approach, IEE Proceedings Control Theory and Applications 151: 125129.

Wang, Z., He, H. and Liao, X. (2004). Stability analysis of uncertain neural networks with delay, Lectures Notes in Computer Science 3173: 44-48.

Wei, J. and Ruan, S. (1999). Stability and bifurcation in a neural network model with two delays, Physica $D$ 130: $255-272$.

Wu, M., He, Y., She, J.-H. and Liu, G.-P. (2004). Delaydependent criteria for robust stability of time-varying delay systems, Automatica 40: 1435-1439.

Ye, H., Michel, A. N. and Wang, K. (1994). Global stability and local stability of Hopfield neural networks with delays, Physics Reviews E 50: 4206-4213.

Zeng, Z., Wang, J. and Liao, X. (2005). Global asymptotic stability and global exponential stability of neural networks with unbounded time-varying delays, IEEE Transactions on Circuits and Systems-II: Express Briefs 52(3): 168-173.

Zhang, J. (2003). Global stability analysis in delayed cellular neural networks, Computers and Mathematics with Applications 45: 1707-1720.

Zhang, Q., Wei, X. and Xu, J. (2003). Global asymptotic stability of Hopfield neural networks with transmission delays, Physics Letters A 318: 399-405.

Zhang, Y. (1996). Global exponential stability and periodic solutions of delay Hopfield neural networks, International Journal of System Sciences 27: 227-231. 